\title{
Perspectivas de la escuela tradicional, nueva y contemporánea
}

\author{
Perspectives of the traditional, new and contemporary school \\ María de la Luz Espindola Juarez ${ }^{a}$, Rafael Granillo Macías ${ }^{b}$
}

\begin{abstract}
:
The different educational paradigms that have emerged over the decades have faced different changes according to the needs of society, in particular of students, teachers and thematic content, for the benefit of our nation. Within this work, some characteristics, authors, advantages and disadvantages of each of the schools, traditional, new and contemporary, can be highlighted.

It is important that we can compare each of these approaches, with which, in addition to knowing about the background, we will know the effective teaching strategies of each of these paradigms, along with the role of the teacher and the student. The main actors of each approach were also addressed, allowing to have an overview of the traditional school, the new school and the contemporary school. This article shows an overview of the different schools and their projection in the different environments in which they are implemented, offering a vision towards the theory. It is also concluded that, although in the traditional school there are elements that could be functional, such as the development of expository discourse in the teacher or favor the cognitive process of memory in students, there are disadvantages and it is less functional for a student to solve real problems compared to other existing models.
\end{abstract}

\section{Keywords:}

Traditional school, new school, compemporary school.

\section{Resumen:}

Los diferentes paradigmas educativos que han surgido a través de décadas, han enfrentado diferentes cambios de acuerdo a las necesidades de la sociedad, en particular de los alumnos, docentes y contenidos temáticos, para beneficio de nuestra nación. Dentro de este trabajo, se pueden destacar algunas características, autores, ventajas y desventajas de cada una de las escuelas, tradicional, nueva y contemporánea.

Es importante comparar cada uno de estos enfoques, con los cuales, además de conocer acerca de los antecedentes, conoceremos las estrategias de enseñanza eficaces de cada uno de estos paradigmas, junto con el rol del docente y del alumno. También se abordaron los principales actores de cada enfoque, permitiendo tener un panorama general de la escuela tradicional, la escuela nueva y la escuela contemporánea. Este articulo muestra un panorama general sobre las diferentes escuelas y su proyección en los diferentes entornos sobre los que se implementan, ofreciendo una visión hacia la teoría. Se concluye también que, aunque en la escuela tradicional existen elementos que podrían resultar funcionales, como el desarrollo de discurso expositivo en el docente o favorecer el proceso cognitivo de la memoria en los estudiantes, existen desventajas y resulta menos funcional para que un estudiante pueda resolver problemas reales en comparación con otros modelos existentes.

\section{Palabras Clave:}

Escuela tradicional, escuela nueva, escuela contemporanea

\footnotetext{
a Autor de Correspondencia, Universidad del Valle de México, Campus Puebla. Email: psicologia_mary1914@ hotmail.com

b Universidad Autómona del Estado de Hidalgo, Ingenieria Industrial, Escuela Superior de Ciudad Sahagún, https://orcid.org/0000-00021015-667X, Email: rafaelgm@uaeh.edu.mx
} 


\section{Introducción}

Desde los inicios de los sistemas educativos, prevalecian dos características esenciales: enseñar religión y mantener los conocimientos de las tradiciones del lugar. En estos inicios las escuelas enseñaban no sólo religión, sino también los principios de la Escritura, Ciencias, Matemáticas y Arquitectura y la mayor parte de la educación estaba en manos de sacerdotes. Es en el siglo XVII donde existió un rápido progreso de muchas ciencias por el intercambio de ideas y de información científica y cultural entre los estudiosos de los diferentes países (Beltran \& Cuellar, 2014).

Actualmente cuando hablamos de educación debemos tomar en cuenta que esta influye de manera significativa en el avance y progreso de nosotros, como personas y como sociedad. La educación no solo nos provee conocimientos, sino que nos permite enriquecer la cultura, el espíritu, los valores y todo aquello que nos caracteriza como seres humanos. En México, la educación es uno de los derechos sociales establecidos en la Constitución que puede considerarse como uno de los grandes avances éticos de nuestra sociedad, ya que su objetivo principal es en beneficio de nuestra nación (Serrano et al, 2017).

Es importante destacar que la educación juega un papel muy importante para el desarrollo, sin embargo, en estas últimas décadas ha adquirido mayor relevancia en el mundo, donde nos enfrentamos diariamente a transformaciones, motivadas en la parte de la ciencia y las tecnologías de la información.

Para comprender mejor estas transformaciones, en este trabajo se presentan las diferentes influencias, enfoques y paradigmas que han existido a partir de los enfoques de la Escuela Tradicional, la Escuela Nueva y la Escuela Contemporánea.

\section{Revisión Teórica de la escuela tradicional, nueva y contemporanea}

\section{Enfoque Tradicional}

La Escuela Tradicional oscila entre los siglos XVII y XVIII / XIX en donde surge la Escuela Pública en Europa y América Latina. El enfoque de esta escuela es conductual y nos dice que el comportamiento es el resultado de las reacciones adquiridas como respuesta a los estímulos externos. Por lo tanto, observamos autores destacados como Skinner (Day, 2000), con los refuerzos y castigos en el condicionamiento, también destaca Bandura (Yarlaque, 2017), quien nos dice que siguiendo ciertos modelos se aprenden diferentes conductas.

En la escuela tradicional, el docente transmite el saber de manera unilateral, por lo que no conlleva necesariamente al aprendizaje de los estudiantes. En este enfoque tradicional, se reforzaban algunas cuestiones alrededor de la memoria a corto y largo plazo, pero en la mayoría de los casos, el estudiante no lograba generar los aprendizajes significativos y desarrollar las habilidades necesarias para resolver problemas reales. Este tipo de escuela se caracterizaba entonces por únicamente por el conocimiento brindado por el docente y adquirido sólo a través de la memorización po el alumno.

Dentro de esta corriente tampoco se permitía la espontaneidad ni interés del estudiante. El papel como protagonista del docente consistía solamente en dar la información que él poseía a través de su experiencia y del contenido de los libros de texto. El estudiante en la escuela tradicional, únicamente debía recibir la información según la libre voluntad del docente, teniendo poco margen para pensar y expresar sus conocimientos. Se exigía memorización del conocimiento, por lo tanto, el estudiante tenía un rol pasivo. (Carneros \& Murillo, 2017).

En esta escuela tradicional se consideraba al maestro como el guía y mediador entre los modelos y el estudiante, obteniendo como resultado que debían imitar y adaptarse a estas pautas (Pérez et.al, 2016). Aunque la escuela tradicional es un modelo pedagógico que ha recibido duras críticas por otras corrientes de pensamiento, también tiene algunos elementos a favor los cuales son:

1. Exige al profesor gran preparación académica y dominio de la materia.

2. Su finalidad es la conservación del orden de cosas, y para ello el profesor asume el poder y la autoridad como transmisor esencial de conocimientos

3. Pone al servicio de los estudiantes los conocimientos y experiencias de un conocedor de la materia, quien además puede resolver dudas de manera inmediata.

4. Posibilita la creatividad y dinamismo del maestro en el desarrollo de los temas.

5. El método fundamental es el discurso expositivo del profesor, con procedimientos siempre verbalistas, mientras el aprendizaje se reduce a repetir y memorizar. 
6. Favorece los procesos de memoria.

7. Permite el trabajo colaborativo y la participación activa del estudiante

En relación a los aspectos negativos de esta escuela tradicional, se encuentran:

1. La relación pedagógica es maestro-alumno, por lo que no suele manejarse la estructura del equipo de trabajo y no favorece la colaboración, ni la cooperación en los estudiantes. Tampoco se estimula la participación, la creatividad, la curiosidad o la iniciativa en los estudiantes.

2. El docente exige disciplina y obediencia, apropiándose de una imagen impositiva, coercitiva y autoritaria.

3. Se asimilan grandes cantidades de información sin tener en cuenta si surgió un aprendizaje significativo. En esta parte se ha evidenciado que gran parte de los conocimientos adquiridos y memorizados, acaban por olvidarse con el tiempo (Serrano et.al, 2017).

4. La acción del alumno está limitada a la palabra del docente y a repetirla, conformando una personalidad pasiva y dependiente.

5. No suele existir convivencia entre la teoría y la práctica.

6. Los contenidos se ofrecen como segmentos fragmentados, posiblemente desvinculados de la realidad.

7. No se controla cómo ocurre el proceso de aprendizaje. Se evalúan resultados y a un nivel reproductivo.

\section{Escuela Nueva}

Dentro de la siguiente etapa, la escuela nueva, encontramos cambios significativos dentro de los procesos educativos, ya que este movimiento, desarrollado a partir de los últimos años del siglo XIX nace como una reacción a la escuela tradicional y a las relaciones sociales que dominaban en la época relacionadas con ciertas ideas de educación y sus prácticas (Polo \& Carazo 2018).

Como menciona Ríos (2014), observamos que en este nuevo perfil educativo se construyó en oposición a la escuela tradicional, centrándose en los intereses espontáneos del estudiante y teniendo como objetivo fortalecer su actividad, libertad y autonomía. Esta nueva forma de trabajo resulto ser todo un conjunto de principios en cuanto a la forma de enseñanza, naciendo de las necesidades de los estudiantes, donde poco a poco se fueron ampliando de acuerdo a las nuevas exigencias de la vida social

Los elementos clave de la escuela nueva fue modificar los vicios de la educación tradicional, con el objetivo de definir un nuevo rol a los diferentes participantes del proceso educativo (Beltrán \& Cuellar 2014). La propuesta de la nueva escuela, destaca que el acto educativo debe tratar a cada estudiante según sus aptitudes y los docentes tienen la función de potencializar esas capacidades ante nuevos retos impuestos por nuestra sociedad, la ciencia y la tecnología. En este escenario, el rol del docente es el de auxiliar el proceso de aprendizaje de cada estudiante, cediendo el poder a ellos y descubriendo las necesidades o el interés de estos. Por otro lado, debido a que la sociedad es un sistema complejo, compuesto de múltiples instituciones; la institución escuela se diferencia de otras, ya que esta brinda conocimientos y habilidades que no se pueden adquirir fuera de ella (Baez \& Latorre, (2016).

Dentro de la parte teórica en la educación, el pedagogo Freire (Lucio, 2015), marcó un avance cualitativo en las ciencias de la educación, ya que analizó la problemática educativa desde un punto de vista integral, es decir, analizando aspectos de la diversidad cultural, la concepción antropologica de la cultura y el dialogo como método de conocimiento. Posteriormente surge el enfoque cognitivo enmarcado por la idea de procesos mentales complejos, tal como lo había abordado J. Piaget (Arellano \& Gordón, 2014), décadas atrás. Un punto importante de este enfoque es que intentó oponerse al conductismo, no rechazando su postura, sino más bien integrado sus teorías a este.

Desarrollado por Piaget (Raynaudo \& Peralta, 2017), el enfoque cognitivo explica que el conocimiento era una interpretación activa de los datos de la experiencia por medio de unas estructuras o esquemas previos. Otro autor como Noam Chomsky trabajó con el tópico del lenguaje y explica que existe una estructura mental innata que permite comprender y producir cualquier enunciado en cualquier idioma natural que conocemos (Chomsky \& Gallego, 2020).

\section{Enfoque Contemporanea}

Pasando al enfoque constructivista algunos autores como Eggen y Kauchak (Hernández, 2019), hacen referencia a que las disputas sobre la mejor manera de enseñar han absorbido las energías como educadores desde el comienzo de la educación formal. Sin embargo, de acuerdo al constructivismo, los aprendizajes deben 
empezar en ideas, sean correctas o incorrectas esta es la pieza fundamental para el desarrollo del proceso enseñanza - aprendizaje y es el principal material que los docentes necesitan al momento de comenzar el proceso para crear el conocimiento en los estudiantes. En esta corriente asume que nada viene de nada, es decir, que conocimiento previo da nacimiento a conocimiento nuevo. Entonces la base fundamental de esta teoría es que las ideas logran que el individuo construya su conocimiento con lo que tenga a su disposición en términos de creencias y conocimiento formal.

En la escuela contemporánea, se desarrolla una formación que permite a los estudiantes resolver problemas de diferente índole de forma autónoma, destacando la autorregulacion, la enseñanza estratégica y el aprendizaje colaborativo. Esto significa que los alumnos pueden enfrentar la búsqueda de soluciones, encontrar respuestas y tener control sobre éstas, dado que, en la mayoría de los casos, los problemas que se presentan implican encontrar respuestas nuevas a preguntas también nuevas.

Dentro de la escuela contemporánea, como elementos a favor se encuentran:

1. La comprensión del aprendizaje simbólico se da en situaciones de vida social.

2. Se permite mayor actividad espontaneidad e interés del estudiante.

3. Se debe desarrollar el proceso educativo de acuerdo a las habilidades y capacidades de cada estudiante.

4. El estudiante aprende más acerca del mundo tecnológico y esto le permite ser un consumidor inteligente de esta área, e incluso le permite evaluar y decidir sobre el futuro de la tecnología en general.

5. El estudiante tiene la posibilidad de participar en clase, exponer sus conocimientos y generar su propio aprendizaje.

6. Permitirá desarrollar al estudiante sus propias capacidades a su propio ritmo.

7. Brinda una educación integral cuyo objetivo es prepararlos para la vida.

Por el contrario, la escuela contemporánea tiene también algunos aspectos en contra, como:

1. La educación tiende a fragmentarse en dos niveles. El que corresponde a un nivel socioeconómico alto y otro destinado a las clases populares, más ligado a la escuela pública. Este último aparece calificado como que enseña menos porque tiene que ocuparse principalmente de las carencias de los estudiantes y también de la violencia familiar y otras patologías propias de la vida contemporánea.

2. No considerar las inquietudes y necesidades de los estudiantes en las comunidades.

3. La evaluación en su mayoría de veces es escrita y se basa especialmente en la memorización, por lo tanto, no permite conocer el proceso ni lo que influyó en él.

Uno de los primeros innovadores en el campo de la pedagogía, John Dewey (Feinberg \& Torres, 2014), quien desarrolla algunos preceptos. Este autor, menciona que la vida escolar debía mejorar y estructurar la vida de fuera. Por lo tanto, en la escuela contemporánea, sería como un laboratorio donde los estudiantes estarían formándose para la resolución de conflictos. Considerando que existen potencialidades diferentes y que la escuela debía dar espacio al desarrollo de todas estas potencialidades. Dewey (Llavador, 2016), puso su experiencia en una escuela norteamericana, sin embargo, fue por un periodo de tiempo corto impidiendo obtener resultados concretos.

\section{Discusion}

Sin duda una tarea de vita importancia para el gobierno, es el reformular el sistema educativo de manera integral, para un bien común, mejorar la calidad y los procesos de enseñanza, actualizando los planes de estudios y capacitando constantemente a los profesores. En Mexico, como en la mayoría de países, se requiere de generar nuevas estrategias que podría incluir un aprendizaje basado en las capacidades de aprender bajo enfoques relacionados con la escuela tradicional y contemporánea, para desarrollar un proceso de aprendizaje que pueda proveer todos los recursos para la adquisición de conocimientos que se adapten a las nuevas necesidades que un mundo globalizado y moderno requiere. La labor del docente, es la construcción de un bien común, aprovechando todas las ventajas que ofrece el actual sistema de gobierno. La educación se posiciona como un factor relevante para conseguir mejores oportunidades laborales y así construir un futuro más próspero, y no sólo para tener mejor calidad de vida, también para ayudar al crecimiento de nuestro país.

En las economías modernas, el conocimiento se ha convertido en uno de los factores más importantes de la producción y las sociedades que más han avanzado en lo económico y en lo social, mismas que han logrado cimentar su progreso en el conocimiento, tanto el que se 
transmite con la escolarización, como el que se genera a través de la investigación en educación, la ciencia y la innovación tecnológica.

\section{Conclusiones}

Es necesario una formación continua de los docentes, no solo dentro de nuestra área (matemáticas, historia, física, psicología, química), sino también en el área pedagógica, ética y psicológica, para poder brindar aquella formación integral que nuestros estudiantes necesitan para enfrentarse al mundo real. Todo ello para favorecer a los estudiantes, al sistema educativo y a la sociedad actual en la que nos encontramos.

Sin duda, la escuela contemporánea, representa la posibilidad de ejercer estrategias de enseñanza que permitan al estudiante no solo generar su propio aprendizaje, sino que le permite desarrollar sus habilidades a su propio ritmo para después poder resolver problemas del mundo real. Aunque en la escuela tradicional existen elementos que podrían resultar funcionales, como el desarrollo de discurso expositivo en el docente o favorecer el proceso cognitivo de la memoria en los estudiantes, definitivamente tiene más desventajas y resulta menos funcional para que un estudiante pueda resolver problemas reales.

\section{Referencias}

Arellano, A. S., \& Gordón, J. (2014). Esbozo crítico sobre las estructuras cognitivas: génesis del pensamiento científico. Sophia: colección de filosofía de la educación, (16), 71-82.

Baez Abarzúa, E., \& Latorre Yáñez, P. (2016). La escuela como institución educativa y su relación con la diversidad de estudiantes con necesidades educativas especiales (Doctoral dissertation, Universidad Academia de Humanismo Cristiano).

Beltrán, R. R., \& Cuellar, M. Y. C. (2014). La modernización de los contenidos y métodos de enseñanza: reflexiones sobre la Escuela Nueva en Colombia. Revista Historia de la Educación Latinoamericana, 16(22), 157172 .

Carneros, S., \& Murillo, F. J. (2017). Aportaciones de las escuelas alternativas a la justicia social y ambiental: Autoconcepto, autoestima y respeto. REICE. Revista Iberoamericana sobre Calidad, Eficacia y Cambio en Educación, 15(3), 129-150.

Chomsky, N., \& Gallego, Á. J. (2020). La facultad humana del lenguaje. Un objeto biológico, una ventana hacia la mente y un puente entre disciplinas. Revista Española de Lingüística, 50(1), 7-34.
Day, R. K. (2000). Skinner y el condicionamiento operante. Los principios de la mejora del rendimiento: Modelos para el aprendizaje en la organización, p. 38 .

Feinberg, W., \& Torres, C. A. (2014). Democracia y educación: John Dewey y Paulo Freire. Cuestiones Pedagógicas, 23, 29-42.

Hernández-Hernández, Z. (2019). Aproximaciones didácticas a la construcción del problema de investigación. Eduscientia, 2(3), 3-15.

Llavador, J. B. (2016). Las variedades de la experiencia educativa: la Escuela-Laboratorio de Dewey. In Democracia y Educación en el siglo XXI. La obra de John Dewey 100 años después: libro de actas del XVI Congreso Nacional y VII Congreso Iberoamericano de Pedagogía [celebrado del] 28 al 30 de junio de 2016, Facultad de Educación, Universidad Complutense de Madrid (p. 151). Sociedad Española de Pedagogía.

Lucio-Villegas, E. (2015). Paulo Freire: La educación como instrumento para la justicia social. Revista Internacional de Educación para la Justicia Social.

Pérez, Á. A., Gelves, B. B. A., Colmenárez, M. A. F. C., \& Ramírez, T. E. C. (2016). Una aproximación a las pedagogías alternativas. Educere, 20(66), 237-247.

Polo, M. Á. C., \& Carazo, P. D. M. (2018). Las corrientes y modelos pedagógicos en el contexto universitario: breves comentarios de su evolución. Portal de las Palabras, 4, 46-52.

Raynaudo, G., \& Peralta, O. (2017). Cambio conceptual: una mirada desde las teorías de Piaget y Vygotsky. Liberabit, 23(1), 110-122.

Ríos Beltran, R. (2014). Escuela Nueva y saber pedagógico en Colombia: apropiación, modernidad y métodos de enseñanza. Primera mitad del siglo XX. Historia y Sociedad, vol. 24, ene-jun 2013.

Serrano, M. T. E., Cantú, A. G., \& Vila, I. M. (2017). Solución de Problemas: Estudio evaluativo de tres enfoques pedagógicos en las escuelas mexicanas. Electronic Journal of Research in Education Psychology, 1(2), 79-96.

Yarlaque Mori, M. M. (2017). Propuesta de Estrategias de Habilidades Sociales basada en la Teoría del Aprendizaje Social de Bandura, para Mejorar las Relaciones Interpersonales en las Estudiantes Universitarias de la Especialidad de Educación Inicial-Lemm-Fachse-Unprg-2017. 